\title{
Anterior Displacement of Ulnar Nerve at the Elbow in Children Based on Ultrasonography
}

\author{
H Shamsul, MS (Ortho)*, A Saw, FRCS*, G John, FRCS ** \\ * Department of Orthopaedic Surgery, University Malaya Medical Centre, Kuala Lumpur, Malaysia \\ ** Department of Radiology, University Malaya Medical Centre, Kuala Lumpur, Malaysia
}

\begin{abstract}
This study involved evaluation of the elbows of 50 children between 4 to 14 years of age. Clinical examination for elbow flexion, extension, range of motion and carrying angle were performed, followed by ultrasonographic examination to determine position of the ulnar nerve in relation to the medial epicondyle upon elbow flexion and extension. Thirty-one elbows showed anterior displacement of the ulnar nerve, of which 14 were due to subluxation and 17 to dislocation. We found that anterior translation of ulnar nerve is present in the elbows up to $31 \%$ of children, especially those with increased range of elbow motion.
\end{abstract}

Key Words:

Ulnar nerve, Instability, Elbow, Children, Ultrasonography

\section{INTRODUCTION}

Ulnar nerve is generally located posterior to the medial epicondyle of the humerus at the elbow. It then enters the flexor compartment of the forearm by passing between the two heads of the flexor carpi ulnaris. In some cases, the nerve either lies anterior to the medial epicondyle ${ }^{1}$ or translates anteriorly whenever the elbow is flexed ${ }^{2}$. Based on clinical examination by palpation on 1000 healthy individuals with 2000 elbows, Childress ${ }^{3}$ reported 162 cases $(16.2 \%)$ of anterior displacement of ulnar nerve when the elbow was flexed. Zaltz ${ }^{4}$ and colleagues performed a similar study on 164 healthy children and reported 33(10\%) ulnar nerves dislocated anteriorly during elbow flexion. More recently, Okamoto et al ${ }^{5}$ examined 200 normal elbows using ultrasonography and found that in 53 elbows $(27 \%)$, the ulnar nerve moved to the tip of medial epicondyle when the elbow was flexed and in another 39 elbows (20\%), the nerve dislocated anteriorly.

Closed reduction followed by percutaneous pin fixation is the most common treatment for suparacondylar fractures of the distal humerus in children ${ }^{6,7}$. Cross wire fixation of both the medial and lateral bone columns remains the most common fixation method despite a significant rate of iatrogenic ulnar nerve injury reported that varies from 2 to $15 \% \%^{8,9}$. Knowing the exact location of ulnar nerve at the elbow may help a clinician to avoid this complication. We therefore undertook this study to investigate the rate and degree of anterior displacement of ulnar nerve at the elbow in children.

\section{MATERIALS AND METHODS}

Fifty healthy children between 4 to 14 years of age were recruited for this study. These children were either being treated for lower limb injuries or were healthy siblings of children coming for follow up in the paediatric orthopaedic clinic. Children who had a history of fracture or severe soft tissue injury to the upper limbs, who were suffering from any systemic illnesses or connective tissue disorders and who had hypermobile joints were excluded. Ethical approval was obtained from the institution before the study was conducted.

Clinical measurement for the range of elbow flexion, extension, total range of elbow motion and carrying angle were obtained using a goniometer. Elbow extension beyond zero degree flexion was described as hyperextension.

For ultrasonographic examination of the elbow, the child initially sat upright on a chair with 30 degrees flexion of the shoulder joint, full extension of the elbow joint and 90 degrees supination of the forearm. The medial epicondyle and olecranon were identified by palpation and ultrasonographic visualization. The transducer was then placed in the transverse plane over the posteromedial aspect of the elbow, with the superior pole of the transducer over the medial epicondyle and the inferior pole over the olecranon process (Fig. 1a). The elbow joint was then passively flexed from 0 to 90 degrees while the transducer remained stationary in relation to the medial epicondyle. Imaging was carried out in the same image plane as elbow extension (Fig. 1b). Imaging of the contralateral elbow was then performed in the same manner. The ulnar nerve was identified on the 
Table I: Frequency of ulnar nerve displacement among groups of carrying angle and degree of ulnar nerve displacement

\begin{tabular}{|lcccc|}
\hline \multirow{2}{*}{ Carrying angle } & \multicolumn{3}{c|}{$\begin{array}{c}\text { Degree of ulnar nerve displacement } \\
\text { Anterior displacement }\end{array}$} \\
\cline { 2 - 5 } & No displacement & Subluxated & Dislocated & Total \\
\hline 0 - 11 degrees & 38 & 11 & 10 & 56 \\
$11-20$ degrees & 31 & 3 & 17 & 44 \\
Total & 69 & 14 & 100 & 17 \\
\hline
\end{tabular}

Table II: Analysis of range of motion and degree of ulnar nerve displacement

\begin{tabular}{|c|c|c|c|c|}
\hline \multirow[t]{2}{*}{ Full range of elbow motion } & \multicolumn{3}{|c|}{$\begin{array}{c}\text { Degree of ulnar nerve displacement } \\
\text { Anterior displacement }\end{array}$} & \multirow[b]{2}{*}{ Total } \\
\hline & No/minimal & Subluxated & Dislocated & \\
\hline 125 to 134 degrees & 32 & 6 & 4 & 42 \\
\hline 135 to 144 degrees & 21 & 3 & 4 & 28 \\
\hline 145 to 154 degrees & 16 & 5 & 5 & 26 \\
\hline 155 degrees or more & 0 & 0 & 4 & 4 \\
\hline Total & 69 & 14 & 17 & 100 \\
\hline
\end{tabular}

basis of its parallel hypoechoic neuronal fascicles separated by hyperechoic connective stroma. This nerve also produces a speckled echogenicity that appears hypoechoic relative to the surrounding hyperechoic fat in an imaging plane transverse to the ulnar nerve (Fig. 2a). The normal speckled appearance of the ulnar nerve may be less apparent if the ultrasound beam is not perpendicular to the nerve fibres due to anisotropy. Identification of the ulnar nerve was then confirmed by scanning proximal and distal to the cubital tunnel and noting the expected location and echotexture of the ulnar nerve.

A diagnosis of ulnar nerve subluxation was made if the ulnar nerve moved to the tip of medial epicondyle on elbow flexion (Fig. 2b). The apex of the medial epicondyle was defined as the point where the two nearly flat cortical surfaces or the humerus formed an angle of approximately 80 - 90 degrees. A diagnosis of ulnar nerve dislocation was made when the ulnar nerve displaced anteriorly beyond the apex of the medial epicondyle (Fig. 2c).

We used ANOVA test to statistically evaluate the correlation between the position of ulnar nerves and the range of motion at the elbow. Chi-Square test was used to determine the correlation between the position of ulnar nerve and other demographic and clinical parameters: age, race, carrying angle of the elbow, elbow hyperextension and elbow flexion. All statistical analyses were calculated using SPSS software program version 11.0 for Windows.

\section{RESULTS}

Out of the 50 children (100 elbows examined), 24 were boys (48\%) and 26 were girls (52\%). Racial makeup was as follows: 25, Malays (50\%); 8 , Chinese (16\%); 13, Indians $(26 \%)$; and 4 , other races $(8 \%)$. The mean age was 8.2 years with a range from 4 to 14 years. The degree of elbow flexion ranged from 115 degrees to 140 degrees with the mean at 131 degrees. The degree of elbow hyperextension ranged from 0 to 20 degrees with the mean at 11 degrees. The range of elbow motion was between 120 and 155 degrees with the mean at 137 degrees. The carrying angle of the elbows ranged from 0 to 20 degrees with the mean at 12 degrees.

The mean carrying angle was 11 degrees for boys and 13 degrees for girls. The mean carrying angle for Malays, Chinese, Indians and other races are 12, 12, 12 and 9 degrees respectively. Statistical analysis using ANOVA demonstrated that there was not a statistically significant ( $\mathrm{p}$ value $>0.05$ ) correlation between sex, race and age and carrying angle of the elbow.

Out of the 100 elbows examined, 31 showed anterior displacement of ulnar nerves on elbow flexion. There were $17(17 \%)$ dislocated and 14 (14\%) subluxated ulnar nerves. Of the 50 children examined, the ulnar nerves of 18 children demonstrated anterior displacement: 13 with bilateral involvement and 5 with unilateral involvement.

Seventeen out of 48 (35\%) elbows in boys and 14 out of 52 (27\%) elbows in girls showed anterior displacement of ulnar nerves. There were 7 subluxations and 10 dislocations in boys versus 7 subluxations and 17 dislocations in girls. There was no statistically significant correlation ( $p$ value $>0.05$ ) between sex and displacement of ulnar nerve.

Age distribution of the children was not uniform due to small sample size. We divided the children into 3 age groups: group one (50 elbows) for those between 4 and 7 years, group two (34 elbows) between 8 to 11 years, group three (16 elbows) 11 years or older. Thirteen out of 50 in group one showed anterior displacement, 14 out of 34 in group two, and 4 out of 16 in group three showed anterior displacement. Again, there was no statistically significant correlation 
108472 NV-OA8 pg29-33.qxd 11/05/2007 08:07 PM Page $31 \bigcirc$ (Black plate)

Anterior Displacement of Ulnar Nerve at the Elbow in Children Based on Ultrasonography

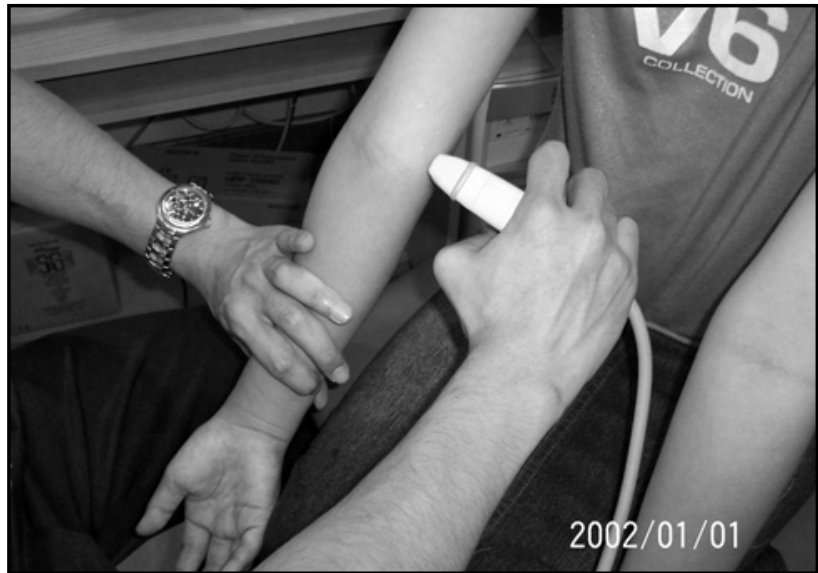

Fig. 1a: Ultrasonography of the ulnar nerve with elbow extension.

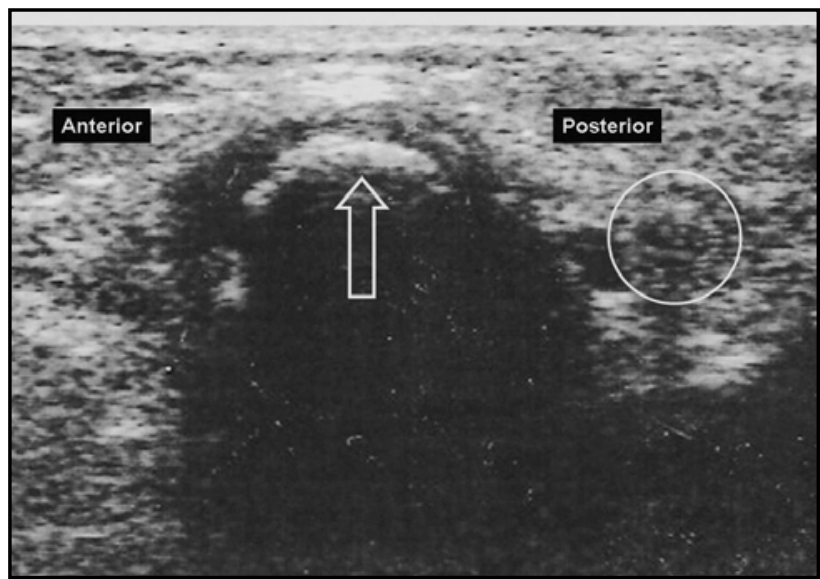

Fig. 2a: Axial image of the ulnar nerve (circle) lying posterior to the tip of medial epicondyle (arrow) in elbow extension.

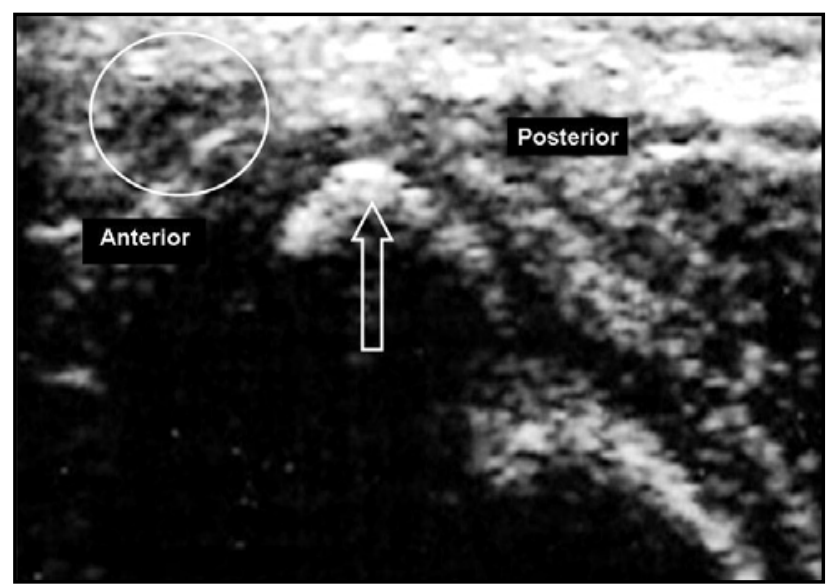

Fig. 2c: Axial image of the dislocated ulnar nerve (circle) lying anterior to the medial epicondyle (arrow) on elbow flexion.

between ( $p$ value $>0.05)$ between age or age groups and anterior displacement of ulnar nerve.

The carrying angle of the elbow was classified into 2 groups: group one, 11 degrees or less and group two, more than 11

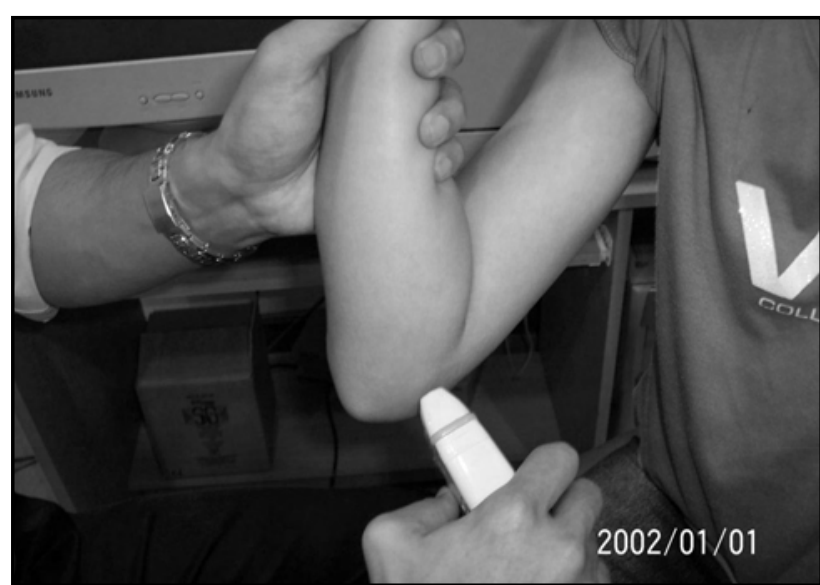

Fig. 1b: Position of transducer in elbow flexion.

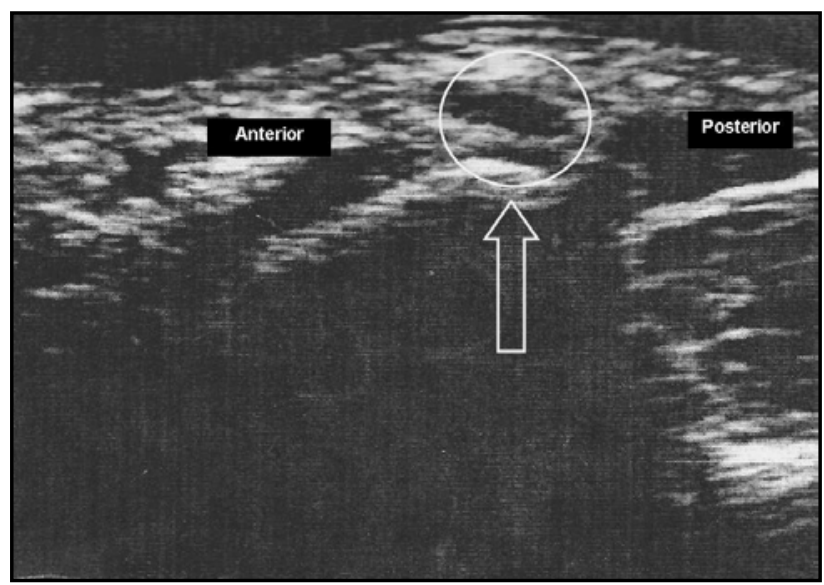

Fig. 2b: Axial image of the subluxated ulnar nerve (circle) lying at the tip of medial epicondyle (arrow) on elbow flexion.

degrees. We also looked into the rate of subluxation and dislocation of the various groups, and again found no statistically significant correlation ( $p$ value $>0.05$ ) between carrying angle group and nerve displacement. (Table I )

Next, we compared elbow flexion and elbow hyperextension to anterior displacement but were unable to show any significant correlation. We then looked at the range of elbow motion, dividing all possible results into 4 groups. In group one (total range of motion between 125 and 134 degrees), 10 out of $42(24 \%)$ elbows showed anterior displacement. In group two (range of motion between 135 and 144 degrees), 7 out of $28(25 \%)$ elbows showed anterior displacement. Results for group three (range of motion between 145 and 154 degrees demonstrated that 10 out of $26(39 \%)$ elbows showed anterior displacement, whereas analysis of group four (155 degrees and more range of elbow motion) found that 4 elbows showed anterior displacement (Table II). Although there is an increased tendency for anterior displacement of ulnar nerve in elbows with higher range of motion, it was not statistically significant ( $p$ value $>0.05$ ). Similarly, statistical analysis showed significant relation $(\mathrm{p}$ 
value $<0.05$ ) between the degree of displacement and total range of motion.

\section{DISCUSSION}

There were no significant difference in the carrying angle between boys and girls as normally observed in adults. Most children in this study have not reached puberty and have not developed this secondary sexual characteristic ${ }^{11}$.

Based on our results, $31 \%$ of elbows in healthy children demonstrated some degree of anterior displacement of the ulnar nerve during passive flexion of the joint. This rate is higher than those reported in studies by Childress ${ }^{3}$ and Zaltz ${ }^{4}$ which were based on clinical palpation. Ultrasonography is generally more sensitive and specific in detecting displacement of peripheral nerves since it allows direct visualization of the structures being observed.

On the other hand, the study on adult elbows by Okamoto ${ }^{5}$ reported a higher rate of ulnar nerve displacement. In adults, the medial epicondyle of distal humerus will be more developed, and distance between ulnar nerve and axis of elbow rotation will be bigger. This increases the excursion of ulnar nerve during elbow flexion, resulting in a tendency to cross the axis towards the anterior aspect of this bony prominence. Furthermore, soft tissue anchoring of the nerve proximal and distal to the elbow may be more rigid in the adults and therefore might not allow sliding of the nerve. All these factors may explain the higher incidence of anterior displacement of ulnar nerve in adults.

Except for total range of elbow motion, we found no statistically significant correlation between age, sex or other physical characteristics of the elbow and increased risk of anterior displacement of the ulnar nerve. Bilateral ulnar nerve displacement was noted in 13 of the 18 children (72\%).
Similar findings were reported by Zaltz et $a l^{4}$ where except for one case, all cases had bilateral ulnar nerve displacement.

In a supracondylar fracture of the distal humerus, flexion of the elbow is necessary to achieve and maintain reduction of the distal fragment before fixation with wires. Wilkins et al had report that though placement of the medial pin directly through the apex of medial epicondyle is a critical, it poses a risk of injuring the ulnar nerve if the nerve is mobile ${ }^{12}$. Based on this study, clinicians should be aware that in children, about one third of ulnar nerves will be located either at the tip or anterior to the medial epicondyle when the elbow is in flexion. Lateral pin fixation is probably a safer option. Foead et $a l^{7}$ demonstrated that in clinical practise, 2 lateral pin fixation technique is as effective as cross medial lateral pin fixation in maintaining reduction of supracondylar fractures of distal humerus in children. It may even be possible to use ultrasonography to locate the ulnar nerve of the injured elbow but this concept requires further evaluation before it can be generally recommended.

\section{CONCLUSION}

Thirty-one per cent of elbows in healthy children have anterior displacement of ulnar nerve during elbow flexion, $17 \%$ of which are dislocated and $14 \%$ are subluxated. Risk of displacement is higher in those with increased range of elbow motion. Measures should be taken to avoid iatrogenic injury of the mobile ulnar nerve during wire fixation for supracondylar fracture of humerus in children.

\section{ACKNOWLEDGEMENT}

We would like to thank Dr. K L Quek and Dr. Farizah Mohd Hairi from the Department of Social and Preventive Medicine for their help in the statistical analysis of this study.

\section{REFERENCES}

1. Tourtas CP and Bergman RA. Anatomic variations of the upper extremity. Churchill Livingstone 1993, 230.

2. Apfelberg DB, Larson SJ. Dynamic anatomy of the ulnar nerve at the elbow. Plastic Recons Surg. 1973; 51: 76-81.

3. Childress HM. Recurrent ulnar nerve dislocation at the elbow . Clin Orthop. 1975; 108: 168-73.

4. Zaltz A, Waters PM and Kasser JR. Ulnar nerve instability in children. J Paed Orthop. 1996; 16: 567-9.

5. Okamoto M, Abe M, Shirai H, Ueda N. Morphology and dynamics of the ulnar nerve in the cubital tunnel. Observation by ultrasonography. J Hand Surg Br. 2000; 25: 85-9.

6. Arino VL, Lluch EE, Ramirez AM, Ferrer J, Rodriguez L, Baixauli F. Percutaneous fixation of supracondylar fractures of the humerus in children. J Bone Joint Surg. 1977; 59A: 914-6. 
Anterior Displacement of Ulnar Nerve at the Elbow in Children Based on Ultrasonography

7. Foead AI, Penafort R, Saw Aik, Sengupta S. Comparison of two methods of percutaneous pin fixation in displaced supracondylar fractures of the humerus in children. J Orthop Surg. 2004; 12: 1, 76-82.

8. Royce RO, Dutkowsky JP, Kasser JR and Rand FR. Neurologic complications after k-wire fixation of supracondylar humerus fractures in children. J Paed Orthop. 1991; 11: 191-4.

9. Brown IC, Zinar DM. Traumatic and iatrogenic neurological complications after supracondylar humerus fractures in children. $J$ Paed Orthop. 1995; 15: 440-3.

10. Rasool MN. Ulnar nerve injury after k-wire fixation of supracondylar humerus fractures in children. J Paed Orthop. 1998; 18: 686-90.

11. Adam DB, Latih MJ, Andrew SR, Joseph DZ. Elbow joint biomechanic: Basic science and clinical application. Orthopedics. 2000; 23(12): 1293-303.

12. Wilkins, KE. Fractures in Children. 3rd edition published by J. B. Lippincott Company 1991. 